\title{
Mobiluncus mulieris
}

National Cancer Institute

\section{Source}

National Cancer Institute. Mobiluncus mulieris. NCI Thesaurus. Code C86519.

A species of anaerobic, Gram positive, rod shaped bacterium assigned to the phylum Actinobacteria. This species is motile by one to eight flagella from multiple origins, oxidase and catalase negative, does not hydrolyze esculin, is stimulated from arginine, weakly reduces nitrate and produces acid from glycogen. M. mulieris is found in the vaginal tract and is a causative agent of vaginitis. 